\title{
Exploring variations in the fundamental constants with ELTs: the CODEX spectrograph on OWL
}

\author{
Paolo Molaro ${ }^{1,2}$, Michael T. Murphy ${ }^{3}$ \\ and Sergei A. Levshakov ${ }^{4}$ for the CODEX team $\dagger$ \\ ${ }^{1}$ Osservatorio Astronomico di Trieste, Via G. B. Tiepolo 11, 34131 Trieste, Italy \\ ${ }^{2}$ Observatoire de Paris 61, avenue de l'Observatoire, 75014 Paris, France \\ ${ }^{3}$ Institute of Astronomy, University of Cambridge, Madingley Road, Cambridge CB3 0HA, UK \\ ${ }^{4}$ Department of Theoretical Astrophysics, Ioffe Physico-Technical Institute, 194021 \\ St.Petersburg, Russia
}

\begin{abstract}
Cosmological variations in the fine structure constant, $\alpha$, can be probed through precise velocity measurements of metallic absorption lines from intervening gas clouds seen in spectra of distant quasars. Data from the Keck/HIRES instrument support a variation in $\alpha$ of 6 parts per million. Such a variation would have profound implications, possibly providing a window into the extra spatial dimensions required by unified theories such as string/M-theory. However, recent results from VLT/UVES suggest no variation in $\alpha$. The COsmic Dynamics EXperiment (CODEX) spectrograph currently being designed for the ESO OWL telescope (Pasquini et al. $2005)$ with a resolution high enough to properly resolve even the narrowest of metallic absorption lines, $R>150000$, will achieve a 2-to-3 order-of-magnitude precision increase in $\Delta \alpha / \alpha$. This will rival the precision available from the Oklo natural fission reactor and upcoming satellite-borne atomic clock experiments. Given the vital constraints on fundamental physics possible, the ELT community must consider such a high-resolution optical spectrograph like CODEX.
\end{abstract}

Keywords. Cosmology: observations, Quasars: absorption lines

\section{Introduction}

Experimental exploration of possible space-time variations in fundamental constants has focused on the dimensionless fine-structure constant, $\alpha=\frac{e^{2}}{\hbar c},=1 / 137.03599911(46)$. In quantum electrodynamics, $\alpha$ plays the role of a coupling constant, representing the strength of the interaction between electrons and photons. It cannot be predicted by theory, and it is one of the twenty-odd "external parameters" in the Standard Model of particle physics.

Many modern theories predict variations in various fundamental constants (e.g. see review in Uzan 2003). String theory implies supersymmetry and predicts six undiscovered dimensions of space. In higher dimensional theories the constants' values are defined in the full dimensional space, and any evolution either in time or space in their sizes would lead to dynamical constants in the 4D space. Varying $\alpha$ is obtained by arbitrarily coupling photon to scalar fields. Scalar fields provide negative pressure and may drive the cosmological acceleration (Bento et al. 2004, Fujii 2005). If the scalar fields are extremely

$\dagger$ L. Pasquini, H. Dekker, S. Cristiani, F. Pepe, M. Haehnelt, G. Avila, B. Delabre, S. D'Odorico, J. Liske, P. Shaver, P. Bonifacio, S. Borgani, V. D'Odorico, E. Vanzella, M. Dessauges-Zavadsky C. Lovis, M. Mayor, M. Viel, F. Bouchy, A. Grazian, L. Moscardini, T. Wicklind, S. Zucker. 
light they could produce variations of constants only on cosmological time scales. The functional dependence of the gauge-coupling constants on cosmological time is not known and even oscillations might be possible during the course of the cosmological evolution (Marciano 1984). In this regard astronomical observations are the only way to test such predictions at different space-time coordinates.

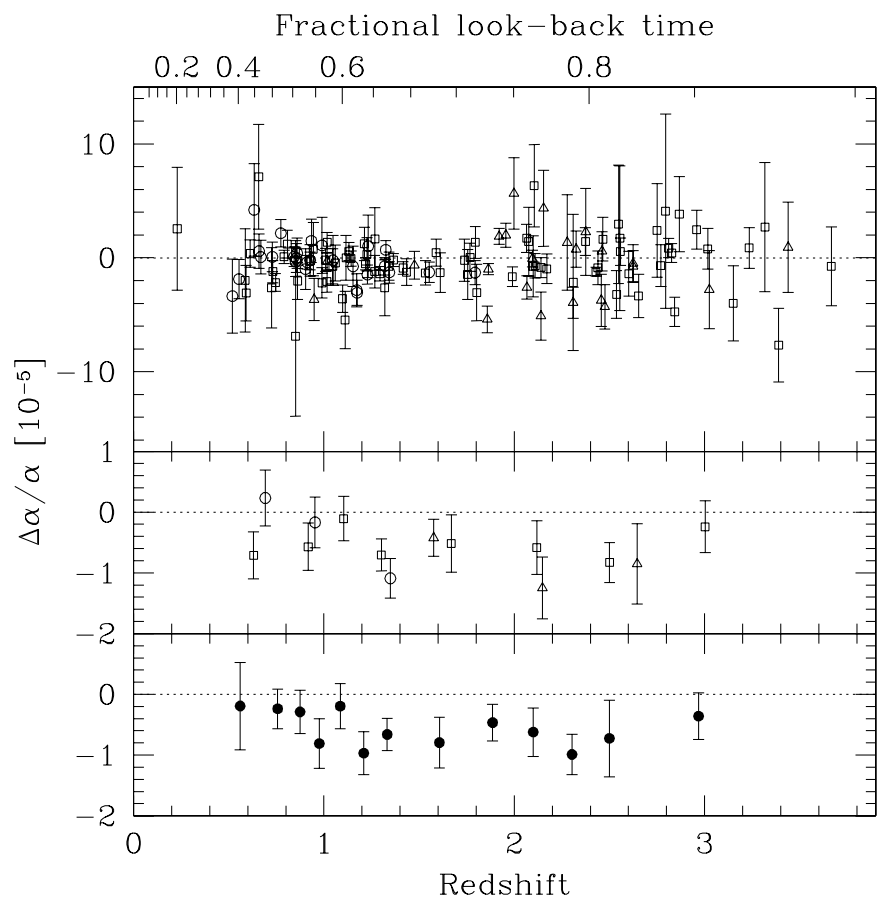

Figure 1. Murphy et al. 2004. The variation in $\alpha$ is found at a $5 \sigma$ confidence level.

\section{Measures and constraints on variability}

Big Bang nucleosynthesis and the cosmic microwave background power spectrum constrains $\Delta \alpha / \alpha=\left(\alpha_{z}-\alpha_{0}\right) / \alpha_{0} \leqslant 10^{-2}$ at $\mathrm{z} \approx 10^{10}$ and at $\mathrm{z} \approx 1100$, respectively. Atomic clocks in laboratory experiments restrict the time-dependence of $\alpha$ at the level of $(\dot{\alpha} / \alpha)_{t_{0}} \approx 10^{-15} \mathrm{yr}^{-1}$, or $|\Delta \alpha / \alpha|<10^{-5}$, for a time of $t \sim 10^{10} \mathrm{yr}$ assuming $\alpha_{z}$ changing linearly with time. The ACES (Atomic Clock Ensemble in Space) project foreseen to fly on the International Space Station in 2007, will operate a cold atom clock in microgravity to test General Relativity and search for a possible drift of the fine structure constant reaching the $10^{-17} \mathrm{yr}^{-1}$ stability range with a gain of two orders of magnitude with respect to present laboratory constraints. Meteoritic data on the radioactive $\beta$-decay of ${ }^{187}$ Re place a bound around $\Delta \alpha / \alpha \leqslant 10^{-7}$ (Olive et al. 2004), and a recent analysis of the isotopic abundance in the Oklo samples provides a hint for a variation although at a very low level: $\Delta \alpha / \alpha \geqslant 4.5 \cdot 10^{-8}$ (Lamoreaux \& Torgerson, 2004).

High resolution spectroscopy of absorption systems lying along the lines-of-sight to background QSOs followed two approaches. One focused on the alkali-doublets (AD) since the comparison between AD separations seen in absorption systems with those measured in the laboratory provides a simple probe of variation of $\alpha$. The current more stringent constraints come from analysis of Si IV absorption systems in 15 systems with 


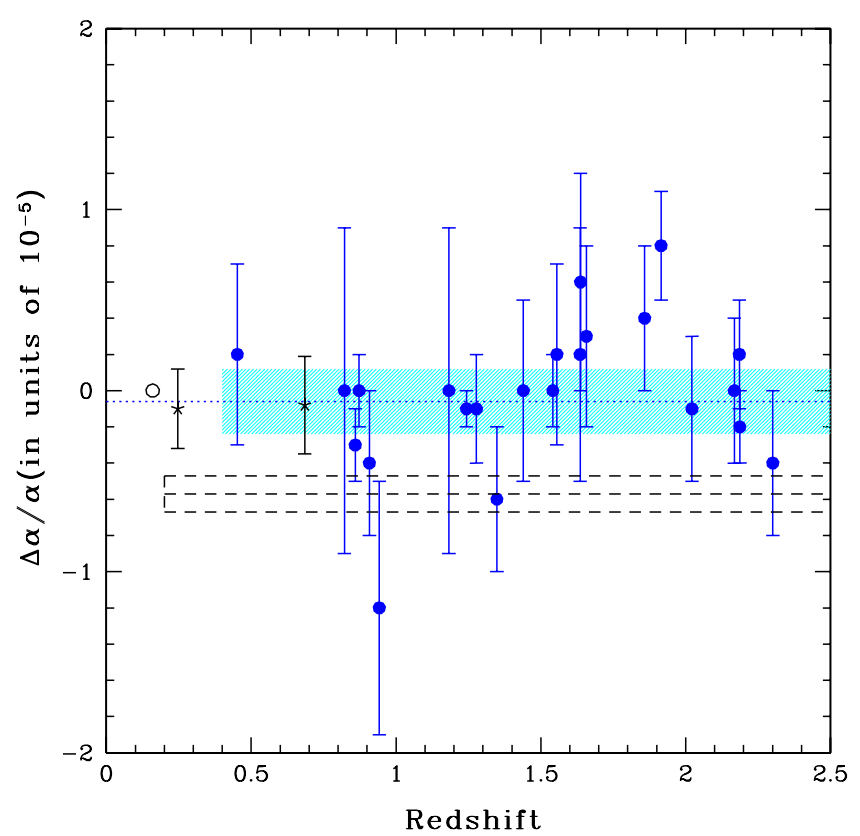

Figure 2. Chand et al. (2004) measures in 23 absorption systems with VLT/UVES. The Keck results are also shown with the dashed line rectangle.

redshift $1.6<z_{a b s}<3: \Delta \alpha / \alpha=(1.5 \pm 4.3) 10^{-6}$ (Chand et al. 2005). A second approach, the multi multiplet (MM) one, compares the line shifts of the species particularly sensitive to a change in $\alpha$ such as $\mathrm{Cr}, \mathrm{Fe}, \mathrm{Ni}$ and $\mathrm{Zn}$ to one with a comparatively minor sensitivity, such as $\mathrm{Mg}$, Si and $\mathrm{Al}$ which is referred to as an anchor (Dzuba, Flambaum \& Webb 1999 and Webb et al. 1999). The sensitivity of each line transition on a change in $\alpha$ is expressed by a coefficient $q$ (Dzuba et al. 1999, 2002).

Applied to Keck/HIRES QSO absorption spectra the MM method has yielded the first evidence for a varying $\alpha$ by Webb et al. (1999), becoming stronger with successively larger samples. The most recent value $\Delta \alpha / \alpha=(-5.7 \pm 1.1) 10^{-6}$ comes from the analysis of 143 absorption systems over the range $0.2<z_{a b s}<4.2$ and is shown in Fig. 1 (Murphy et al. 2004). The deduced variation in $\alpha$ at about $5 \sigma$ confidence level and its implications are extraordinary. However, the result has not been confirmed by other authors. Chand et al. (2004) have analyzed $23 \mathrm{Mg}$,Fe absorption systems in higher signal-to-noise ratio $(\mathrm{S} / \mathrm{N})$ spectra from a different telescope and spectrograph, the VLT/UVES, claiming a null result over the range $0.4<z_{a b s}<2.3 \Delta \alpha / \alpha=(-0.6 \pm 0.6) 10^{-6}$, which is shown in Fig. 2. A variant of this methodology makes use only of pairs of FeII lines which differ significantly in their $q$ values, in order to minimize potential systematic errors. This has been applied in a couple of systems, at $z_{a b s}=1.839$ in the spectrum of Q1101-264 and at $z_{a b s}=1.15$ in the spectrum of HE0515-4414, providing $\Delta \alpha / \alpha=\left(0.4 \pm 1.5_{\text {stat }}\right) \times 10^{-6}$ and $\Delta \alpha / \alpha=\left(-0.07 \pm 0.84_{\text {stat }}\right) \times 10^{-6}$, respectively (Levshakov et al. 2005, 2006a). The discrepancy between the VLT/UVES and Keck/HIRES results is yet to be resolved and demands for a high resolution spectrograph at an ELT for significant progress.

It is also interesting to note that Fujii (2005) has been able to fit both the Chand et al. and the Levshakov et al. null results by means of an oscillatory behaviour for a varying $\alpha$. A behaviour which can even be traced by eye in the original data of Chand et al. shown 
in Fig. 2. To probe such an intriguing possibility, very high precision measurements at individual redshifts are required. Moreover, the MM measures which use $\mathrm{Mg}$ as an anchor relay on terrestrial relative composition of the $\mathrm{Mg}$ isotopes. Since the frequency shifts of $\Delta \alpha / \alpha$ are of the order of magnitude of the typical isotope shifts, a departure from these values can produce different results for the measures which take $\mathrm{Mg}$ as an anchor. Sub-solar ${ }^{25,26} \mathrm{Mg} /{ }^{24} \mathrm{Mg}$ ratios would make a variation of $\alpha$ even more significant. Suggestion that this my be the case comes indirectly from a recent upper limit in the ${ }^{13} \mathrm{C}$ abundance $\left({ }^{12} \mathrm{C} /{ }^{13} \mathrm{C}>200,1 \sigma\right)$, in the system at $z_{a b s}=1.15$ in the spectrum of HE0515-4414 (Levshakov et al. 2006b). Since both ${ }^{25,26} \mathrm{Mg}$ and ${ }^{13} \mathrm{C}$ are produced in the Hot Bottom Burning stage of AGBs, a low ${ }^{13} \mathrm{C}$ imply possibly low ${ }^{25,26} \mathrm{Mg}$. In the case of the Chand et al. data set the relaxation of this assumption would have produced a $\Delta \alpha / \alpha=\left(-3.6 \pm 0.6_{\text {stat }}\right) \times 10^{-6}$. This illustrates well the fact that the case for variability requires a better understanding of the isotopic evolution of the absorption clouds, a problem which can be addressed only with a spectrograph of very high resolution able to separate the isotopic lines such as the one foreseen for CODEX.

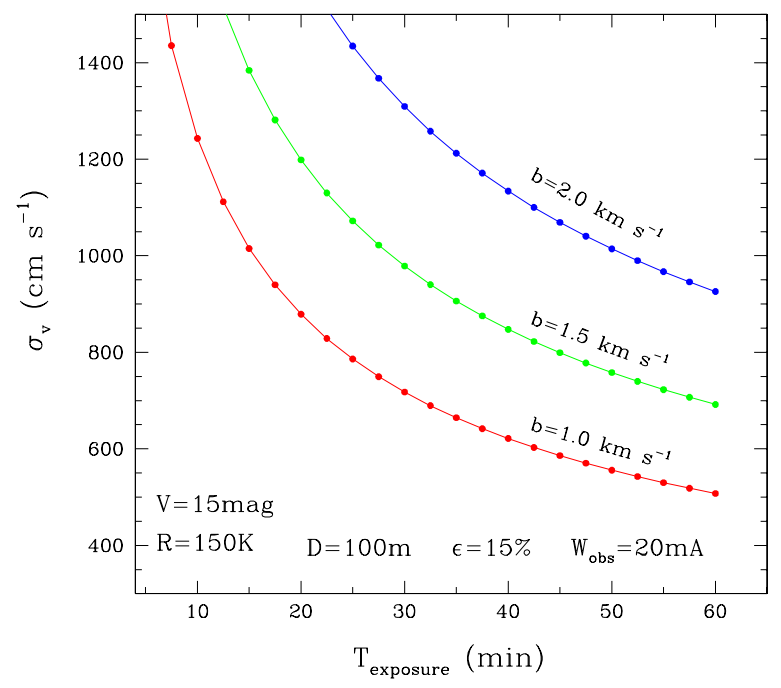

Figure 3. Wavelength precision measurement of a single metal line with broadening of 1, 1.5 and $2 \mathrm{~km} \mathrm{~s}^{-1}$, with a spectrograph with $\mathrm{R}=150000$ at a $100 \mathrm{~m}$ telescope. See text for details.

\section{With CODEX on OWL}

A measurement of $\Delta \alpha / \alpha$ is essentially a measurement of the wavelength for a pair or more lines with different sensitivity coefficients. Therefore the accuracy of a $\Delta \alpha / \alpha$ measurement is ultimately determined by the precision with which a line position can be determined in the spectrum. With current spectrographs with $\mathrm{R}=\lambda / \delta \lambda \approx 4 \cdot 10^{4}$ the observed line positions can be known with the accuracy of about $\sigma_{\lambda} \approx 1 \mathrm{~m} \AA$ (or $\Delta v=$ $60 \mathrm{~m} \mathrm{~s}^{-1}$ at $5000 \AA$ ), then $\tilde{\sigma}_{\Delta \alpha / \alpha} \simeq 10^{-5}$ for a typical pair. This value is normally further improved to reach one part per million when more transitions and/or more systems 
are available. Any improvement with respect to this figure is related to the possibility to measure line positions more accurately. This can be achieved with an increase in the resolving power of the spectrograph up to the point in which the narrowest lines formed in intervening physical clouds are resolved, and with an increase of the signal-tonoise ratio in the spectrum (Bohlin et al. 1983). The metal lines which are observed in the QSO absorption systems have intrinsic widths of a few $\mathrm{km} \mathrm{s}^{-1}$, rarely of less than $1 \mathrm{~km} \mathrm{~s}^{-1}$. The CODEX spectrograph with a resolving power $\mathrm{R} \approx 150000$ matches well these requirements.

In Fig. 3 we show the precision in velocity measurement of an absorption line as a function of exposure time with the OWL telescope. We used the Bohlin et al. formula for an absorption line with an equivalent width of $0.02 \AA$ and $b$ values of $1,1.5$ and $2 \mathrm{~km} \mathrm{~s}^{-1}$. The spectrograph is the CODEX one as described in Pasquini et al. (2006) and a global efficiency of $15 \%$ is assumed. A precision of $5-10 \mathrm{~m} \mathrm{~s}^{-1}$ is obtained in a relatively short time of 1 hour. This would imply a precision in the determination of $\alpha$ of the order of $\tilde{\sigma}_{\Delta \alpha / \alpha} \simeq 10^{-7}$ for a single pair of lines. The precision can then further be increased by extending the exposure time or by statistics using other lines belonging to the same system or more systems with similar redshift. The overall final precision will rival, and probably surpass, the one available from the Oklo natural fission reactor and upcoming satellite-borne atomic clock experiments. Given the deep insights on several aspects of fundamental physics and cosmology, the ELT community must consider such a high-resolution optical spectrograph like CODEX.

\section{Acknowledgements}

We acknowledge the contribution of the whole CODEX team.

\section{References}

Bento, M.C., Bertolami, O. \& Santos, N.M. 2004, PhRvD 70, 730

Bohlin R.C., Jenkins, E.B., Spitzer, L., York, D.G., Hill, J.K., Savage, B.D. \& Snow, T.P. 1983, ApJS 51, 277

Chand, H., Srianand, R., Petitjean, P. \& Aracil, B. 2004, A\&A 417, 853

Chand, H., Petitjean, P., Srianand, R. \& Aracil, B. 2005, A\&A 430, 47

Dzuba, V.A., Flambaum, V.V., Kozlov, M.G. \& Marchenko, M.V. 2002, PhRvA 66, 501

Dzuba, V.A., Flambaum, V.V. \& Webb, J.K. 1999, PhRvA 59, 230

Fujii, Y. 2005, Physics Letters B 616, 141 (astro-ph/0502191)

Lamoreaux, S.K. \& Torgerson, J.R. 2004, PhRvD 691, 1701

Levshakov, S.A., Centurión, M., Molaro, P. \& D’Odorico, S. 2005, A\&3A 434, 827

Levshakov, S.A., Centurión, M., Molaro, P., D’Odorico, S., Reimers, D., Quast, R. \& Pollmann, M. 2006a, these proceedings, astro-ph/051228

Levshakov, S.A., Centurión, M., Molaro, P. \& Kostina, M.V. 2006b, A\& A in press

Marciano, W.J. 1984, Phys. Rev. Lett. 52, 489

Murphy, M.T., Flambaum, V.V., Webb, J.K., Dzuba, V.V., Prochaska, J.X. \& Wolfe, A.M. 2004, in: S.G. Karshenboim \& E. Peik (eds.), Astrophysics, Clocks and Fundamental Constants, (Springer-Verlag: Berlin, Heidelberg), p. 131

Murphy, M.T., Webb, J.K. \& Flambaum, V.V. 2003, MNRAS 345, 609

Olive, K.A., Pospelov, M., Qian, Y-Z., Manhès, G., Vangioni-Flam, E., Coc, A. \& Cassé, M. 2004, PhRvD 69b, 770

Pasquini, L. et al. 2006, these proceedings

Uzan, J.-P. 2003, RvMP 75, 403

Webb, J.K., Flambaum, V.V., Churchill, C.W., Drinkwater, M.J. \& Barrow, J.D. 1999, PhRvL 82,884 


\section{Discussion}

SRIANAND: With the CODEX wavelength range (4000-6000A) it will not be possible to observe many ion transitions to probe the $\alpha$ variability.

Molaro: With the wavelength accuracy foreseen for CODEX $\left(0.1 \mathrm{~ms}^{-1}\right)$ it will not be a problem to observe lines in different frames since the signal is a few $\mathrm{ms}^{-1}$ for $\Delta \alpha / \alpha \sim 10^{-7}$. So it does not seem to be a limitation. 